\title{
Stability limits of an optical frequency standard based on free $\mathrm{Ca}$ atoms
}

\author{
J. A. Sherman and C. W. Oates \\ NIST \\ 325 Broadway \\ Boulder, CO 80027 \\ Email: jeff.sherman@nist.gov
}

\begin{abstract}
We have quantified a short term instability budget for an optical frequency standard based on cold, freely expanding calcium atoms. Such systems are the subject of renewed interest due to their high frequency stability and relative technical simplicity compared to trapped atom optical clocks. By filtering the clock laser light at $657 \mathrm{~nm}$ through a high finesse cavity, we observe a slight reduction in the optical Dick effect caused by aliased local oscillator noise. The ultimately limiting technical noise is measured using a technique that does not rely on a second clock or fs-comb.
\end{abstract}

\section{INTRODUCTION}

Optical frequency standards based on trapped atoms and ions offer unmatched absolute uncertainties [1], [2]. However, a clock based on cold but untrapped atoms may still offer competitive levels of short term stability while remaining technically simpler and more readily commercialized. Here we discuss limits to the stability of an optical standard featuring cold, freely expanding, Ca atoms [3].

Briefly, atoms are collected in a magneto-optical trap (MOT) by use of the strong ${ }^{1} S_{0} \leftrightarrow{ }^{1} P_{1}$ transition at $423 \mathrm{~nm}$. Though this MOT period lasts only $2.8 \mathrm{~ms}$, many atoms are retrapped between clock cycles, yielding a steady-state atom number of order $10^{6}$. An external cavity diode laser at $657 \mathrm{~nm}$, stabilized to a ULE glass Fabry-Perot cavity (linewidth $\sim 9 \mathrm{kHz}$ ), is made resonant with the allowed inter-combination transition ${ }^{1} S_{0} \leftrightarrow{ }^{3} P_{1}$. The MOT is shuttered for $0.44 \mathrm{~ms}$, and a sequence of $657 \mathrm{~nm}$ pulses forms a Ramsey-Bordé atom interferometer [4] in the freely falling and expanding atoms. Counter-propagating, horizontally aligned pairs of Ramsey pulses suppress residual first-order Doppler shifts, enabling high resolution, and high $\mathrm{S} / \mathrm{N}$, spectroscopy [5], [6]. Resonant $423 \mathrm{~nm}$ light is used to measure the atoms' ground state population before and after the clock pulse sequence. Dividing these collected fluorescence signals yields a spectroscopic feature normalized against atom number fluctuations with a linewidth (FWHM) of $\sim 1 \mathrm{kHz}$, and signal-to-noise ratio $(\mathrm{S} / \mathrm{N})$ of about 50 in $3.3 \mathrm{~ms}$. The clock instability limit implied by these results is about $2 \times 10^{-15}(\tau / 1 \mathrm{~s})^{-1 / 2}$; which is roughly consistent with the quantum projection noise limit given a coherent fringe contrast of $\sim 10 \%$.

We measure the $\mathrm{Ca}$ clock instability to be consistently $3-4 \times 10^{-15}(\tau / 1 \mathrm{~s})^{-1 / 2}$ when compared against a superior local optical standard [7] via a fs-comb. We calculate that the

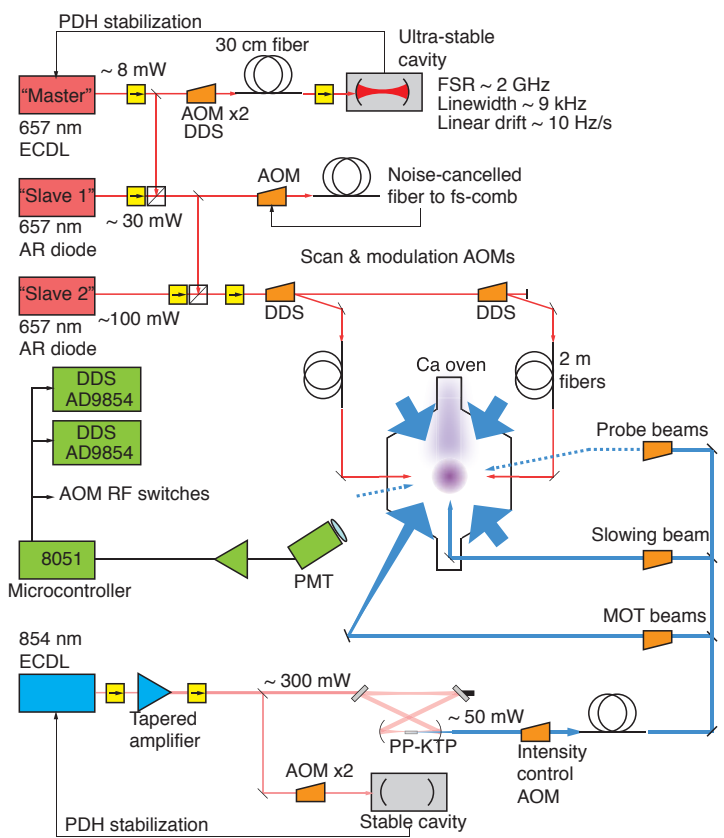

Fig. 1. The free Ca clock is among the simplest optical frequency standards. Slowing, cooling, trapping, and probing radiation at $423 \mathrm{~nm}$ is derived from a frequency-doubled external cavity diode laser. A diode laser at $657 \mathrm{~nm}$, AR-coated and placed in a Littman external cavity, is stabilized to an ultra-high finesse optical cavity. Injection-locked 'slave' laser systems boost the $657 \mathrm{~nm}$ power to enable very short Ramsey pulses and homogenous broadening of the clock transition up to the residual Doppler linewidth of $\sim 2 \mathrm{MHz}$. A microcontroller triggers the clock sequence every $3.3 \mathrm{~ms}$, acquires fluorescence data, and servos the clock laser on the atomic resonance.

optical Dick effect [8], [9] contributes $3.6 \times 10^{-15}(\tau / 1 \mathrm{~s})^{-1 / 2}$ to this instability. About half of the Dick effect is eliminated by filtering the clock laser through a high-finesse optical cavity to remove high frequency local oscillator noise. We measure a comparable amount of instability, $1.8 \times 10^{-15}(\tau / 1 \mathrm{~s})^{-1 / 2}$, due to technical sources. In this paper we discuss these contributions and highlight measurement techniques used to quantify them with minimal use of a second clock or fs-comb.

\section{A. A simplified apparatus}

Figure 1 shows the current version of the apparatus, which has been optimized for stability and fast cycle rate, rather than accuracy. For instance, the MOT gradient magnetic field 


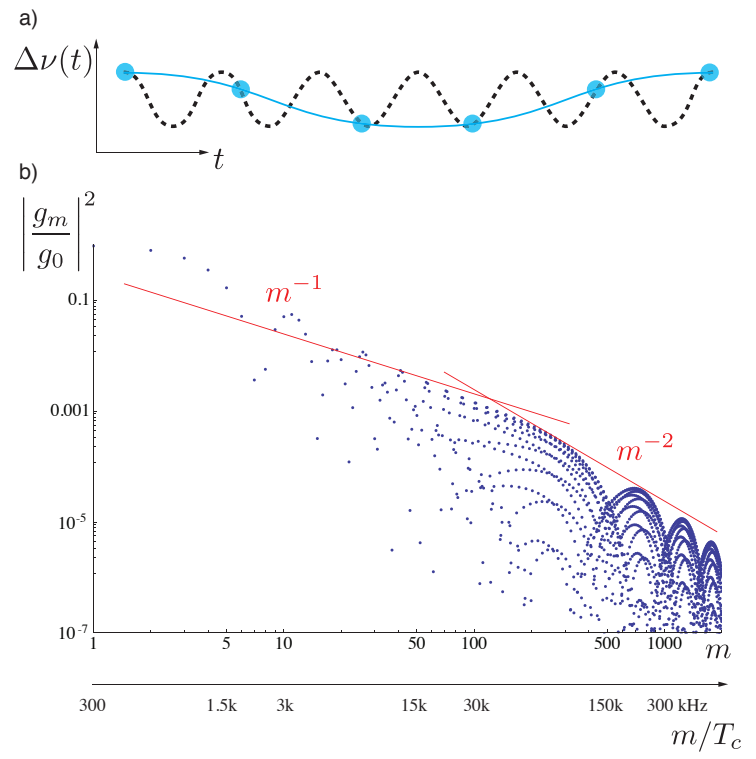

Fig. 2. (a) A cartoon illustrates the optical Dick effect as an aliasing, or downsampling of noise. The dashed line represents a particular Fourier component of laser frequency noise close to the clock measurement repetition rate; the width of each blue circle represents the atomic interrogation period of each clock cycle. Since these interrogation windows are short, it takes many clock cycles to properly average over the noise component. The aliased noise (blue line) is not genuine; if written by a servo onto the local oscillator it can actually increase the short term instability of a clock. (b) Given the RamseyBordé pulse sequence parameters, we calculate sensitivity coefficients $g_{m} / g_{0}$ in Eq. 1 which describe how a local oscillator frequency noise spectrum is aliased into white noise.

remains on permanently, eliminating vibrations that result from rapid toggling [10]. We forgo a quenched second-stage of atom cooling [10] in order to minimize the sequence duration and maximize the atomic interrogation duty cycle. The effusive atomic beam source is located about $15 \mathrm{~cm}$ away from the interaction region; no Zeeman slower or chirped slowing beam is employed. Key acousto-optic modulators between the clock laser and the atoms, and between the laser and the stabilization cavity, are driven with maser referenced DDS synthesizers (labeled DDS in Figure 11. The only fiber optics path that is actively noise-cancelled is the long run to the fs-comb.

\section{LOCAL OSCILLATOR NOISE}

\section{A. The optical Dick effect}

Since the clock's repetition rate is $300 \mathrm{~Hz}$, a digital servo locking the laser to the atomic resonance should strongly suppress local oscillator noise at $1 \mathrm{~s}$ averaging times. However, since the fraction of the clock sequence spent interrogating the atomic resonance - the duty cycle - is small, high frequency fluctuations are not averaged to zero but instead are downsampled. This process, known as the optical Dick effect, results in extra white noise with a fractional instability:

$$
\sigma_{d}^{2}(\tau)=\frac{1}{\tau} \sum_{m=1}^{\infty} S_{y}\left(m / T_{c}\right)\left|\frac{g_{m}}{g_{0}}\right|^{2} .
$$

Local oscillator noise $S_{y}$ at harmonics $m$ of the cycle rate $1 / T_{c}$ are aliased by the imperfect interrogation. Sensitivity
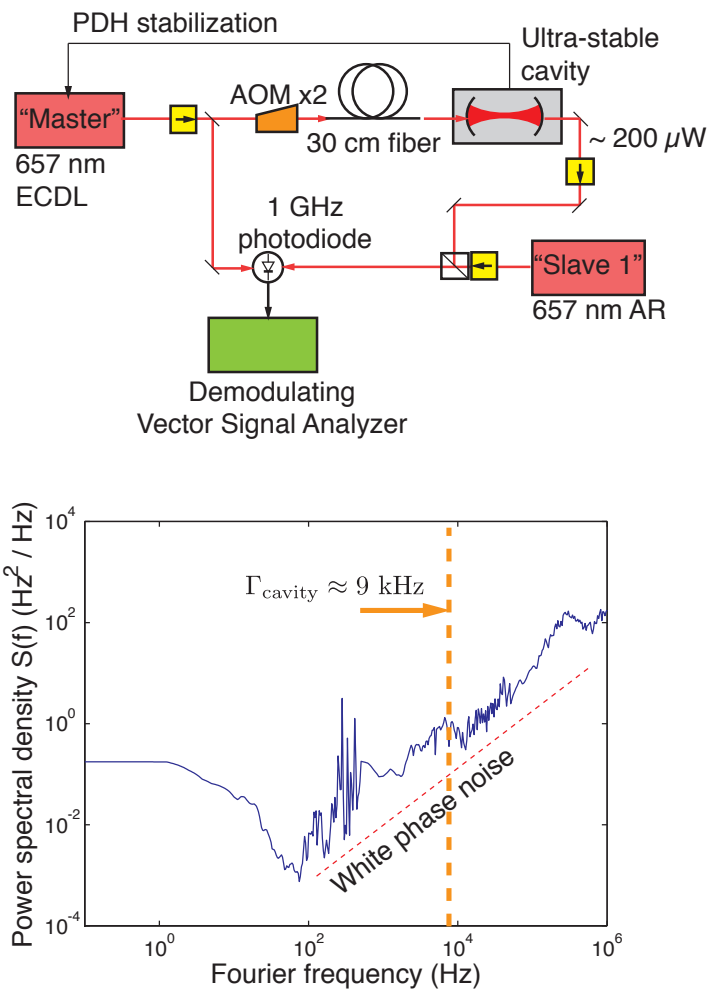

Fig. 3. Fast laser frequency noise is suppressed by filtering through a highfinesse optical cavity; the cavity acts to average out fluctuations faster than the photon ring-down timescale of $\tau=1 / \Gamma_{\text {cavity }} \sim 0.1 \mathrm{~ms}$. To determine the transfer function of the cavity, we heterodyne laser beams before and after transmission through a high finesse optical cavity used for laser stabilization. The noise added by the injection locking to a second laser is independently evaluated.

coefficients,

$$
g_{m} \equiv \frac{1}{T_{c}} \int_{0}^{T_{c}} g(t) e^{-2 \pi i m t / T_{c}} d t, \quad(m>0)
$$

tend to shrink as the interrogation duty cycle approaches $100 \%$ because the sensitivity of the atomic population to oscillator phase $g(t)=2 \delta P /\left.\delta \phi\right|_{\delta_{\phi} \rightarrow 0}$ approaches a constant value. The sensitivity function $g(t)$ for the Ramsey-Bordé pulse sequence is derived elsewhere [8].

Figure 2 illustrates the aliasing effect and shows calculated Dick effect coefficients for our nominal clock cycle having an interrogation duty cycle of $13 \%$. The Ramsey $\pi / 2$ pulses last $T_{R}=0.75 \mu \mathrm{s}$. The duration between Ramsey pulses is $T=215 \mu \mathrm{s}$. The dead-time between pairs of opposing Ramsey pulses is $T_{\nu}=5.8 \mu \mathrm{s}$. Increasing the two Ramsey dark periods lasting $T$ would increase resolution and the interrogation duty cycle, but would also inflict a loss of signal; the lifetime of the excited clock state is $0.4 \mathrm{~ms}$. Numerical analysis of Eq. 1 with our estimated laser noise spectrum $S_{y}$ shows that more than $99 \%$ of the Dick effect instability is due to harmonics $m<100$, or laser noise frequency components $f<30 \mathrm{kHz}$. 


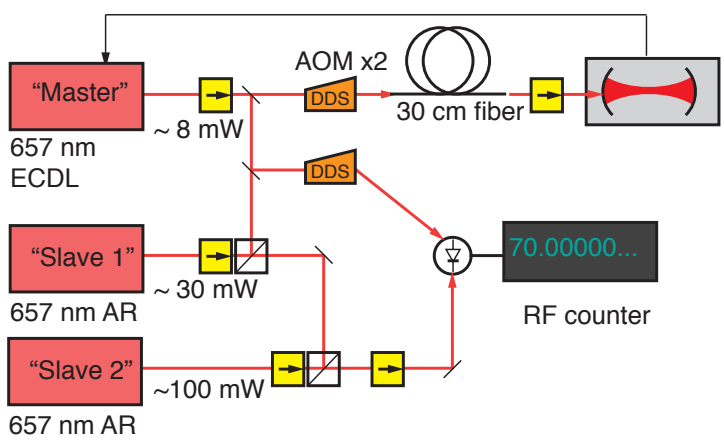

Fig. 4. Illustrated here is a heterodyne between the master external cavity diode laser and an injection-locked 'slave' laser, which is itself seeded by another 'slave' laser. An RF beatnote is created by shifting one of the beams with an acousto-optic modulator (AOM) driven by a maser-referenced synthesizer (labeled DDS). The Allan deviation of maser-referenced RF counter is related to the optical frequency fractional Allan devation relevant to Ca clock operation with $\sigma_{y}(\tau)=\sigma_{\nu}(\tau) / f_{\text {optical }}$. Direct counting of a DDS frequency yields a baseline of $\sigma_{y}(1 \mathrm{~s})=6.0 \times 10^{-18}$. Table II shows the results for several such heterodyne measurements; all instabilities are much lower than the observed clock instability. The noise spectra have not been studied in detail.

\section{B. Clock laser filtering}

We find the instability of the cavity-stabilized clock laser (while unlocked to the atomic resonance) to be $2.5 \times 10^{-15}$ after 1 second of averaging by comparison to a superior optical reference. This instability is consistent with that derived from the laser's noise spectrum from $1 \mathrm{~Hz}-1 \mathrm{kHz}$ found by demodulating the laser's beatnote with a referenced fs-comb. Faster laser noise components, perhaps due to residual phase noise not suppressed by the Pound-Drever-Hall stabilization scheme, can be downsampled by the Dick effect. To examine what fast noise might be suppressed by filtering the clock laser, we heterodyne clock laser beams before and after transmission through the high-finesse stabilization cavity. As shown in Figure 3 . noise rising as $f^{2}$ is observed that contributes directly very little to the local oscillator instability at 1 second; however, through the Dick effect mechanism, the noise above $\Gamma_{\text {cavity }} \approx 9 \mathrm{kHz}$ is responsible for roughly half of the Dick effect instability. The unfiltered and filtered laser Dick effect instability limits are

$$
\begin{aligned}
\sigma_{\mathrm{d},}(\tau) & =3.6 \times 10^{-15}(\tau / 1 \mathrm{~s})^{-1 / 2} \\
\sigma_{\mathrm{d}, \text { filt. }}(\tau) & =2.4 \times 10^{-15}(\tau / 1 \mathrm{~s})^{-1 / 2}
\end{aligned}
$$

We do not see as large an improvement from clock laser filtering as other groups [11] due to our shorter cycle time and larger interrogation duty cycle.

\section{Other noise sources}

We performed heterodyne measurements between the master ECDL beam and several derived laser beams-after injection locking, after short fiber optic runs, after acousto-optic modulation (AOM) - to identify other sources of noise that might contribute to the clock instability. An example arrangement is shown in Figure 4 In each heterodyne experiment, $\mathrm{AOM}$ frequencies and the RF counter were maser referenced.
TABLE I

INSTABILITY CONTRIBUTIONS DETERMINED BY HETERODYNE MEASUREMENTS.

\begin{tabular}{ll}
\hline Heterodyne test & $\sigma_{y}(\tau=1 \mathrm{~s})$ \\
\hline DDS counting baseline & $6.0 \times 10^{-18}$ \\
Before/after cavity & $6.3 \times 10^{-16}$ \\
Before/after 1.5m PM fiber & $1.3 \times 10^{-16}$ \\
Master vs. injection locked 'slave 1' & $1.6 \times 10^{-16}$ \\
Master vs. injection locked 'slave 2' & $1.8 \times 10^{-16}$
\end{tabular}

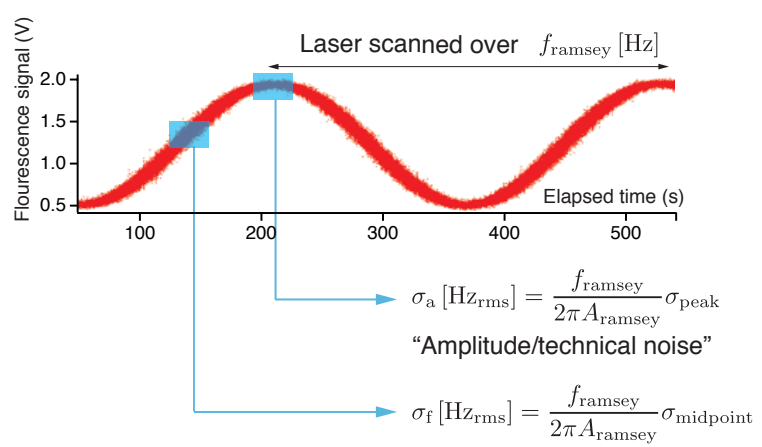

"Total system noise"

Fig. 5. In the absence of a second local oscillator or atomic reference, one can still derive useful absolute instability of a clock by using the atomic reference as a frequency discriminator. Here, the unlocked clock laser is slowly scanned over a Ramsey-Bordé fringe. Point-to-point rms deviations in the fluorescence signal are larger near the frequency-sensitive nodes. Technical noise, which is presumed to be largely laser frequency independent, is observable at the peaks and troughs of the fringe. The clock's operational parameters can be optimized to minimize each noise level. The technical noise level sets a limit on ultimate system stability even in the absence of local oscillator noise. The noise level at the fringe nodes is quantitatively consistent with the overall clock performance when compared against superior optical standards.

Table I expresses these noise contributions as fractional Allan deviations of the optical frequency at 1 second.

\section{TECHNICAL AND TOTAL NOISE EVALUATION}

\section{A. Self-evaluation of instability}

Without the benefit of a second, independent clock laser or atomic reference, one can still evaluate some aspects of the absolute instability of a Ca clock apparatus. With a clock laser detuning $\nu$, frequency fluctuations $\delta \nu(t)$, and Ramsey dark period $T$, the spectroscopy method produces a fluorescence signal [4]:

$$
\rho_{e}(t) \propto A \cos \left(2 \pi \times 4 T \times(\nu+\delta \nu(t))+\delta \rho_{e}(t) .\right.
$$

The laser detuning $\nu$ can be slowly scanned over this feature; laser noise $\delta \nu(t)$ is translated into fluctuations in fluorescence proportional to the local slope $\partial \rho_{e} / \partial \nu$. At the Ramsey fringe nodes, this slope is constant and computable using the Ramsey fringe amplitude $A$ and separation $f_{\text {Ramsey }}=1 /(4 T)$. The rootmean-square deviation of successive measurements of $\rho_{e}$ can be expressed in as a frequency instability by

$$
\sigma_{\nu}\left(\tau=T_{c}\right)[\mathrm{Hz}]=\frac{1}{2 \pi A(4 T)} \sigma_{\rho}\left[V_{\mathrm{rms}}\right] .
$$




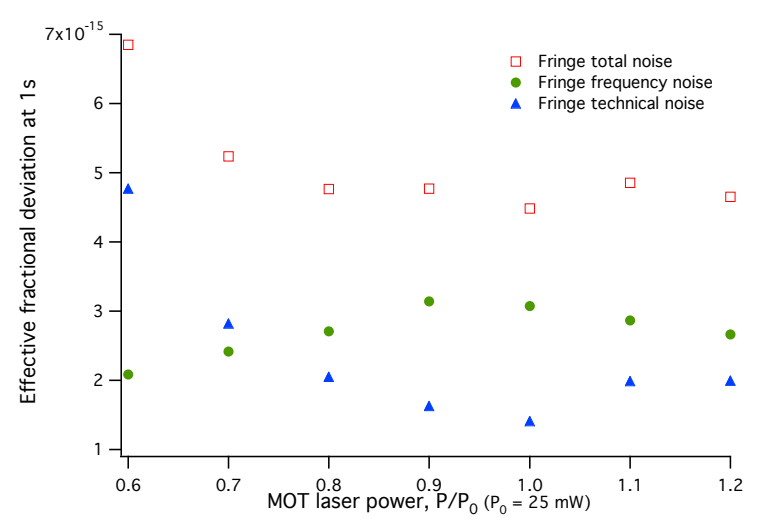

Fig. 6. The instability of the clock system is measured by using the atomic resonance as a frequency discriminator in open loop operation. Noise on the Ramsey fringe peaks and nodes can be related to effective Allan deviations due to technical and frequency-dependent noise, respectively. Here we see how these noises vary as a function of MOT trapping power. An optimum operation condition, the minimum of the total noise, is found without using a second clock or fs-comb.

At the peaks and troughs of the Ramsey fringe we observe technical noise $\delta \rho_{e}(t)$ which is largely independent of the clock laser frequency but nonetheless contributes noise when the servo is locked to a nodal point. At these lock-points, the technical noise will be equivalent to uncorrelated frequency noise using the same conversion factor $1 /(2 \pi A 4 T)$. Several potential sources of technical noise are suppressed by normalizing the signal to atom number, and by dithering the RamseyBordé fringe pattern to remove the incoherent excitation background. The remaining technical noise could include shotto-shot atom number fluctuations imperfectly accounted for by the normalization pulse, fluctuations in $657 \mathrm{~nm}$ pulse power, and frequency noise on the $423 \mathrm{~nm}$ probe pulses. Path length, pointing, and wavefront fluctuations between Ramsey pulses in a single clock cycle also appear as excess technical noise, but are dependent on the laser frequency as well. In general, fluctuations in any systematic effect leading to inaccuracy adds instability as well, though dithering of the Ramsey fringe suppresses such effects varying slower than half the cycle rate.

Fourier transforms of recorded $\rho_{e}(t)$ series at the peaks, toughs, and nodes indicate these noises are largely white from $0.5-150 \mathrm{~Hz}$, and thus have equivalent noise spectra of $S_{\nu}(f)=\sigma_{\nu}^{2} /\left(f_{\text {rep }}\right)$, where $f_{\text {rep }}$ is the clock cycle rate. Effective fractional Allan deviations can be constructed with $\sigma_{y}^{2}(\tau)=\left(S_{\nu} / 2\right)(1 / \tau)\left(1 / f_{\text {optical }}\right)$.

Figure 6 demonstrates the utility of this technique. The MOT laser power is varied around its nominal power of $25 \mathrm{~mW}$, and the effective deviation at $1 \mathrm{~s}$ implied by the open-loop fringe noise is plotted. Technical and frequency noise (corresponding to peak and node noise levels) are separately plotted, and their sum agrees well with the observed clock instability when the unlocked local oscillator noise is subtracted.

\section{CONCLUSion}

Previous efforts have aggressively pursued an uncertainty budget for the $\mathrm{Ca}$ optical frequency standard. In Table III we present instead an instability budget at $1 \mathrm{~s}$ of averaging with no account of ultimate accuracy. We conclude that three different methods of evaluating the instability share good agreement: direct measurement against a superior optical standard, a quadrature sum of the calculated optical Dick effect instability and known technical noise sources, and an open loop measurement using the atoms as a frequency discriminator. The latter technique never relies on a second clock or fs-comb, but it is directly sensitive to the open-loop local oscillator noise.

Another important result is that while filtering the clock laser through our high-finesse cavity decreases the Dick effect limit somewhat, it remains comparable to the technical noise that would ultimately limit the system if the local oscillator were significantly improved. Further study must focus on identifying and reducing the principle sources of technical noise. Since this level is thought to be near that of quantum projection noise, an increase in the number of atoms might be necessary for further improvements.

\section{ACKNOWLEDGMENT}

The authors would like to thank colleagues T. Fortier and S. Diddams for fs-comb measurements, and A. Ludlow, N. Lemke, R. Fox, and Y. Jiang for comparison measurements with the $\mathrm{Yb}$ optical lattice clock and local oscillator. We thank R. Fox, A. Curtis, F. Bondu, G. Wilpers, and Y. LeCoq for their contributions to earlier versions of the $\mathrm{Ca}$ apparatus.

\section{REFERENCES}

[1] H. Katori, "Optical lattice clocks and quantum metrology," Nature Photonics, vol. 5, pp. 203-210, 2011.

[2] C. Chou, D. Hume, J. Koelemeij, D. Wineland, and T. Rosenband, "Frequency comparison of two high-accuracy $\mathrm{Al}^{+}$optical clocks," Physical review letters, vol. 104, no. 7, p. 70802, 2010.

[3] C. Oates, F. Bondu, R. Fox, and L. Hollberg, "A diode-laser optical frequency standard based on laser-cooled Ca atoms: Sub-kilohertz spectroscopy by optical shelving detection," The European Physical Journal D-Atomic, Molecular, Optical and Plasma Physics, vol. 7, no. 3, pp. 449-460, 1999.

[4] C. Bordé, C. Salomon, S. Avrillier, A. Van Lerberghe, C. Bréant, D. Bassi, and G. Scoles, "Optical ramsey fringes with traveling waves," Physical Review A, vol. 30, no. 4, p. 1836, 1984.

[5] G. Wilpers, C. Oates, and L. Hollberg, "Improved uncertainty budget for optical frequency measurements with microkelvin neutral atoms: Results for a high-stability ${ }^{40} \mathrm{Ca}$ optical frequency standard," Applied Physics B: Lasers and Optics, vol. 85, no. 1, pp. 31-44, 2006.

[6] C. Degenhardt, H. Stoehr, C. Lisdat, G. Wilpers, H. Schnatz, B. Lipphardt, T. Nazarova, P. Pottie, U. Sterr, J. Helmcke, et al., "Calcium optical frequency standard with ultracold atoms: Approaching $10^{-15}$ relative uncertainty," Physical Review A, vol. 72, no. 6, p. 062111, 2005.

[7] Y. Jiang, A. Ludlow, N. Lemke, R. Fox, J. Sherman, L. Ma, and C. Oates, "Making optical atomic clocks more stable with $10^{-16}$-level laser stabilization," Nature Photonics, vol. 5, pp. 158-161, 2011.

[8] A. Quessada, R. Kovacich, I. Courtillot, A. Clairon, G. Santarelli, and P. Lemonde, "The dick effect for an optical frequency standard," Journal of Optics B: Quantum and Semiclassical Optics, vol. 5, p. S150, 2003.

[9] G. Dick, "Local oscillator induced instabilities in trapped ion frequency standards," California Institute of Technology, Jet Propulsion Lab, Pasadena, CA, Tech. Rep., 1987. 
TABLE II

A SHORT-TERM INSTABILITY BUDGET FOR A FREE CALCIUM OPTICAL CLOCK.

\begin{tabular}{|c|c|c|c|}
\hline Noise source & Symbol & Measurement technique & $\begin{array}{l}\text { Fractional Allan devi- } \\
\text { sion at } 1 \mathrm{~s}\end{array}$ \\
\hline $\begin{array}{l}\text { Local oscillator, } \\
\text { unlocked }\end{array}$ & $\sigma_{L}$ & $\begin{array}{l}\text { Noise spectrum measured from beat with } \\
\text { Yb-clock on fs-comb }\end{array}$ & $2.5 \times 10^{-15}$ \\
\hline Dick effect, unfiltered & $\sigma_{\mathrm{d}}$ & Calculated & $3.6 \times 10^{-15}$ \\
\hline $\begin{array}{l}\text { Dick effect, filtered } \\
\text { through cavity }\end{array}$ & $\sigma_{\mathrm{d}, \text { filt. }}$ & $\begin{array}{l}\text { Filtered noise obtained by filtered/unfiltered } \\
\text { heterodyne }\end{array}$ & $2.4 \times 10^{-15}$ \\
\hline $\begin{array}{l}\text { Technical noise, and } \\
\text { atom/photon shot } \\
\text { noise }\end{array}$ & $\sigma_{\text {tech. }}$ & $\begin{array}{l}\text { 'Self-evaluated' open-loop Ramsey fringe } \\
\text { noise at peaks/troughs }\end{array}$ & $1.8 \times 10^{-15}$ \\
\hline $\begin{array}{l}\text { Additional noise from } \\
\text { injection locking, } \\
\text { fibers, etc. }\end{array}$ & $\sigma_{\text {add }}$ & Heterodyne measurements & $0.6 \times 10^{-15}$ \\
\hline \multicolumn{3}{|c|}{ Quadrature sum of $\sigma_{\mathrm{d} \text {, filt. }}, \sigma_{\text {tech. }}, \sigma_{\text {add. }}$ (filtered laser case) } & $3.0 \times 10^{-15}$ \\
\hline \multicolumn{3}{|c|}{ Quadrature sum of $\sigma_{\mathrm{d}}, \sigma_{\text {tech. }}, \sigma_{\text {add. }}$ (unfiltered laser case) } & $4.0 \times 10^{-15}$ \\
\hline \multicolumn{3}{|c|}{ Measured instability against $\mathrm{Yb}$ lattice clock, filtered laser, typical } & $3.5(5) \times 10^{-15}$ \\
\hline \multicolumn{3}{|c|}{ 'Self-evaluated' open loop Ramsey fringe noise at nodes, $\sigma_{L}$ subtracted in quadrature } & $3.6 \times 10^{-15}$ \\
\hline
\end{tabular}

[10] G. Wilpers, C. Oates, S. Diddams, A. Bartels, T. Fortier, W. Oskay, J. Bergquist, S. Jefferts, T. Heavner, T. Parker, et al., "Absolute frequency measurement of the neutral 40-Ca optical frequency standard at $657 \mathrm{~nm}$ based on microkelvin atoms," Metrologia, vol. 44, p. 146, 2007.

[11] T. Nazarova, C. Lisdat, F. Riehle, and U. Sterr, "Low-frequency-noise diode laser for atom interferometry," JOSA B, vol. 25 , no. 10 , pp. 1632 $1638,2008$. 